\title{
Estenosis de la apertura piriforme y síndrome de incisivo central único: Casos clínicos
}

\section{Piriform aperture stenosis and solitary median maxillary central incisor: Clinical cases}

Constanza Díaz S ${ }^{1}$, Daniel Retuert², Francisco Krause P³, Rodolfo Nazar S².

\begin{abstract}
RESUMEN
El síndrome incisivo central único es un trastorno que involucra anomalías de la línea media. Se puede presentar con dificultad respiratoria poniendo en peligro la vida del recién nacido, debido a malformaciones nasales. Estas malformaciones incluyen atresia de coanas y la estenosis de la apertura del orificio piriforme. Debe pensarse en esta última en todo recién nacido con estridor y dificultad respiratoria de grado variable, asociado a la dificultad de pasar una sonda a través de la región anterior de las fosas nasales. El diagnóstico se confirma por tomografía computarizada del macizo craneofacial y el examen nasofibroscópico. La conducta terapéutica dependerá de la gravedad de los síntomas, pero en general si es que no hay compromiso respiratorio severo, se prefiere una conducta expectante, y esperar el crecimiento craneofacial del niño, para aumentar permeabilidad nasal.
\end{abstract}

Presentamos dos casos clínicos.

Palabras clave: Obstrucción nasal, estenosis congénita de la apertura piriforme, síndrome de incisivo único central.

Abstract

Solitary median maxillary central incisor syndrome is a rare disorder involving midline abnormalities. It may present with life threatening respiratory distress in the neonate secondary to nasal malformations. These include choanal atresia and pyriform aperture stenosis. The last to be thought in any newborn with stridor and respiratory distress associated with the difficulty of passing a tube through the anterior region of the nostrils. The diagnosis is confirmed by craniofacial CT scan and nasolaryngoscope evaluation. The therapeutic approach depends on the severity of symptoms.

Key words: Solitary median maxillar central incisor síndrome, congenital pyriform apertura stenosis, nasal obstruction.

\footnotetext{
Médico Cirujano, Hospital Fuerza Aérea de Chile

Departamento Otorrinolaringología, Hospital Clínico de la Universidad de Chile

Departamento Otorrinolaringología, Clínica Las Condes.
}

Recibido el 2 de agosto, 2016. Aceptado el 18 de diciembre, 2016. 


\section{INTRODUCCIÓN}

El síndrome incisivo central único (SICU) es una alteración del desarrollo rara y poco frecuente, caracterizada por la presencia de un megaincisivo central y otras anomalías de la línea media asociados. Defectos de la cavidad nasal son bien reconocidos en este síndrome y la atresia de coanas 0 estenosis de la apertura piriforme suele estar presente. Los recién nacidos son respiradores nasales obligados y, por tanto, la obstrucción nasal puede ser potencialmente mortal. La presencia de patologías de la línea media y dificultad respiratoria neonatal debe alertar al médico sobre la posibilidad de síndrome de SICU ${ }^{1,2}$.

La estenosis congénita de la apertura piriforme (ECAP) se origina de forma secundaria al excesivo crecimiento del proceso nasal del maxilar superior de forma bilateral, produciendo una disminución del calibre de la apertura piriforme nasal, con la subsiguiente aparición de signos y síntomas de obstrucción. Se puede asociar a un megaincisivo 0 incisivo central medio solitario maxilar y, también, se han descrito asociaciones con alteraciones endocrinas importantes, como el déficit de hormona de crecimiento, disgenesia tiroidea, hipotiroidismo, episodios de hipoglicemia y ausencia de hipófisis anterior con panhipopituitarismo. El cariotipo de pacientes con ECAP o megaincisivo puede ser anormal, pudiendo presentar alteraciones en los cromosomas 18 y $13^{3}$.
El cuadro clínico depende del grado de estenosis, desde signos leves de insuficiencia respiratoria nasal hasta casos graves que constituyen una verdadera urgencia vital y que requieren intubación orotraqueal, con tratamiento variable, desde una actitud expectante con aplicación de medidas conservadoras hasta cirugía.

\section{CASOS CLÍNICOS}

Se presentan 2 casos clínicos.

El primer caso corresponde a una paciente recién nacida gemelar de pretérmino 36 semanas, sexo femenino. Estudio prenatal sin hallazgos patológicos. En la atención inmediata no se logra atravesar coanas al aspirar secreciones, además presenta dificultad respiratoria con cianosis y retracciones por lo que requiere oxígeno, con lo que cede sintomatología. Es evaluado por equipo de Otorrinolaringología del Hospital Clínico de la Universidad de Chile, se le realiza nasofibroscopía (Figura 1) en fosa nasal izquierda, examen dificultoso, se accede por piso nasal, poco espacio, se logra visualizar coana permeable. En fosa nasal derecha no se logra visualizar coana ya que no se logra sobrepasar nivel de apertura piriforme. Examen impresiona atresia de coana unilateral derecha por lo que se decide realizar TAC de cavidades paranasales sin contraste (Figura 2), donde se visualizan ambas fosas nasales reducidas de

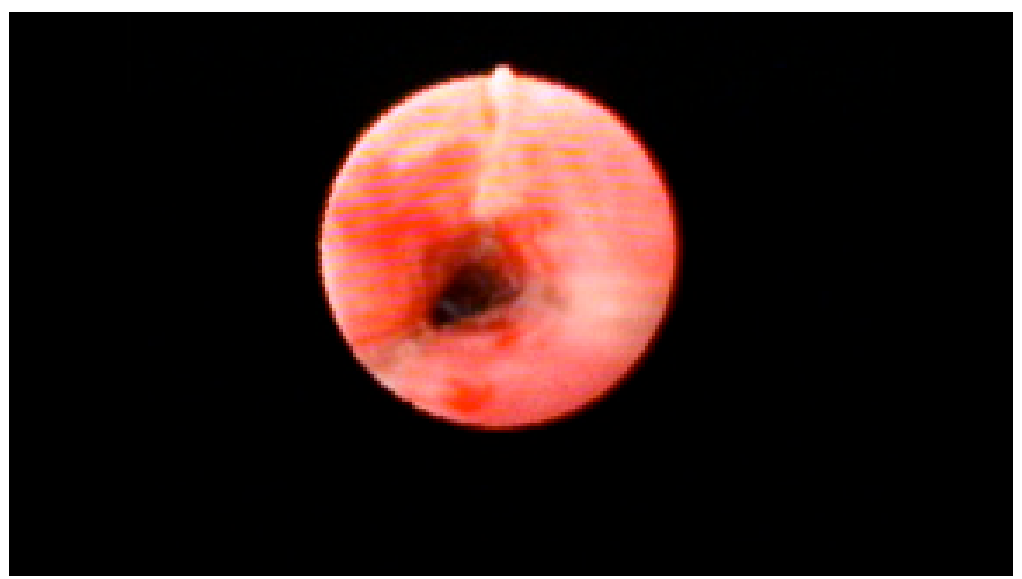

Figura 1. Nasofibroscopía de caso clínico número 1 que evidencia zona de estenosis en región de apertura piriforme por fosa nasal izquierda. 


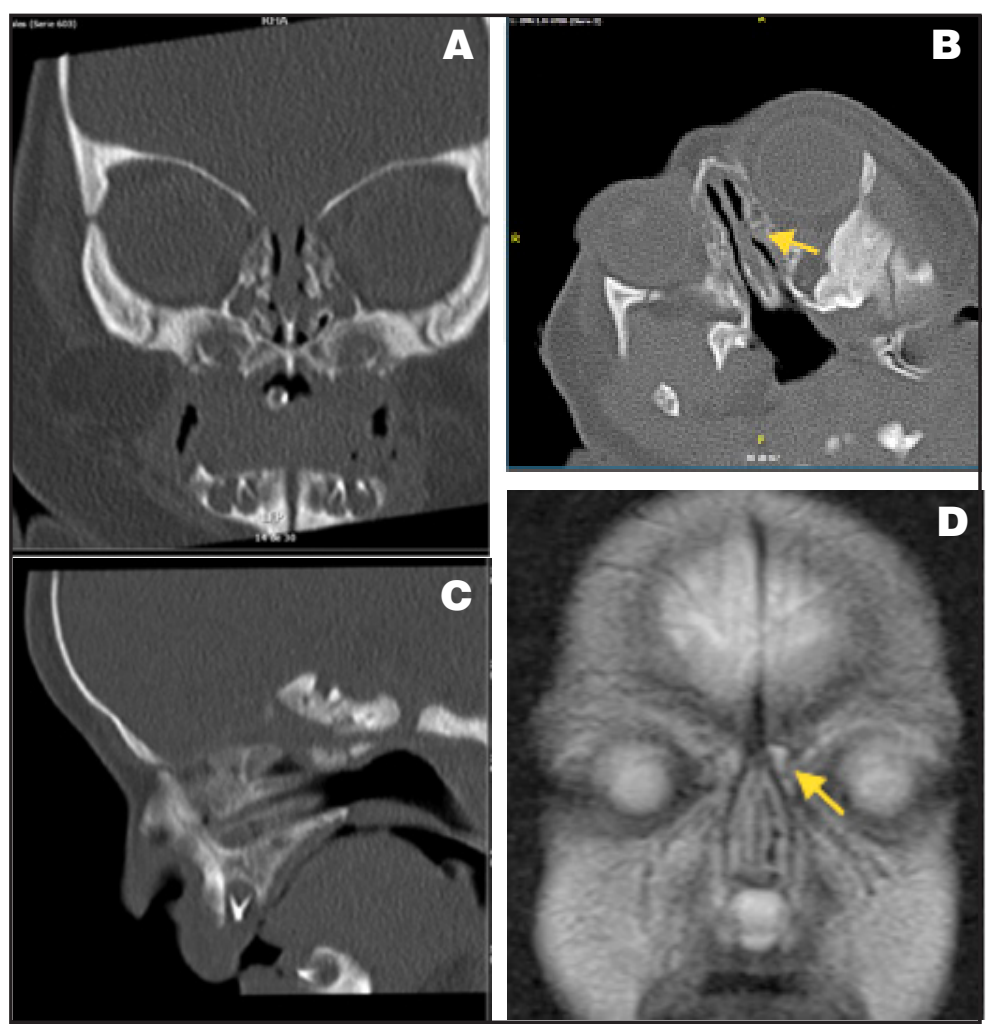

Figura 2. Tomografía computarizada de nariz y cavidades paranasales del caso clínico número 1. a) Corte coronal que evidencia zona de estenosis de fosas nasales. b) Corte axial donde se ve estrechez en la región de la apertura piriforme. c) Corte sagital que evidencia la permeabilidad coanal. d) Corte coronal de la resonancia nuclear magnética donde se evidencia presencia de incisivo central maxilar único.

amplitud y coanas amplias y permeables. Paciente evoluciona con dificultad respiratoria leve en reposo y que aumenta en lactancía por lo cual continúa con oxígeno con mascarilla. RNM de cerebro y cara destaca estrechamiento en amplitud de ambas coanas y de la apertura piriforme bilateral que mide de 1 a 1,5 mm (Figura 2), además presencia de incisivo central maxilar único (Figura 3). Con estos resultados es evaluada por comité rinosinusología del Hospital Clínico de la Universidad de Chile, donde se realiza nasofibroscopía con equipo de 2,7 mm donde se observa en fosa nasal derecha estenosis en zona de apertura piriforme, se logra visualizar coana libre. En fosa nasal izquierda estenosis en zona apertura piriforme, mayor que en lado derecho, se logra pasar zona de estenosis y se visualiza coana libre. A partir del día 3 de vida la dificultad respiratoria disminuye, se decide una conducta expectante y manejo conservador con aseo nasal continuo con Fisiolimp ${ }^{\circledR}$ y control en 3 meses 0 según necesidad con otorrinolaringología. Es dado de alta a los 7 días sin necesidad de oxígeno. Posteriormente asiste a control de los 3 meses con buen incremento pondoestatural y sin dificultad respiratoria.

El segundo caso corresponde a un paciente recién nacido pretérmino de 37 semanas, de sexo femenino, adecuado para la edad gestacional, peso nacimiento $2.780 \mathrm{gr}$. talla $47 \mathrm{~cm}$. Apgar 9-9. Presentó estridor nasal progresivo desde el primer día de vida que se asoció a dificultad en la alimentación y leve dificultad respiratoria, sin cianosis en reposo ni al llanto, sin episodios de desaturación ni requirimientos de oxígeno. Sintomatología cede en decúbito prono y se mantiene con alimentación fraccionada por chupete. Se solicita evaluación por otorrinolaringología que incluyó nasofibroscopía con equipo de 2,7 mm de diámetro que mostró 


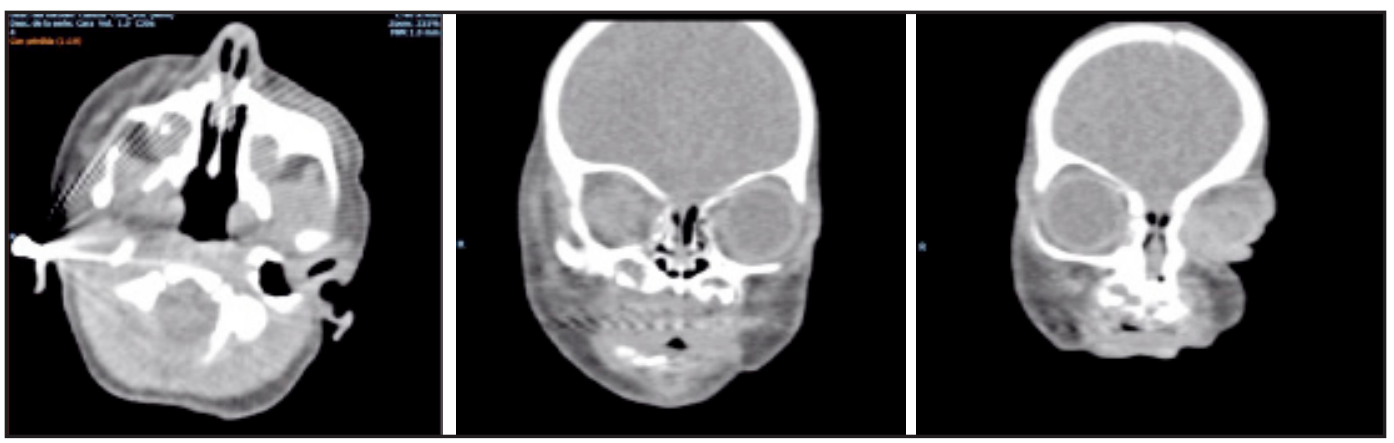

Figura 3. Tomografía computarizada de nariz y cavidades paranasales del caso clínico número 2. a) Tomografía computarizada sin contraste de cavidades paranasales, corte axial. Se observa leve disminución de amplitud en la región posterior a la apertura piriforme. b) Tomografía computarizada. Corte coronal, donde se observa permeabilidad presente pero disminuida en la región de la apertura piriforme.

desviación septal no obstructiva y cornetes hipertróficos, pero se logra demostrar permebilidad descartando atresia o estenosis de coanas. Se solicita tomografía computarizada (TAC) de cara, que informa estrechez de apertura piriforme más acentuada a derecha. Se decide conducta expectante, manejo con mometasona y seguimiento con otorrinolaringología posterior al alta, para observar evolución y evaluar eventual necesidad de cirugía.

Consulta al mes y medio de vida, por congestión nasal a repetición, sin otro síntoma agregado, se deja tratamiento con ceterizina, Nasonex ${ }^{\circledR}$ e Iliadin ${ }^{\circledR}$ SOS en crisis, tras lo cual evoluciona en buenas condiciones con controles periódicos a los 2, 4, 9 meses sin cuadros agudos intercurrentes. Último control con especialidad a los 2,5 años, se observó en TAC de control fosa nasal derecha aún algo estenótica. Paciente evoluciona sin necesidad de cirugía se mantiene tratamiento con levocetirizina y se indica control anual.

\section{DISCUSIÓN}

El síndrome incisivo central único (SICU) es un trastorno del desarrollo raro, caracterizado por la forma y la posición del incisivo central superior única. Puede ocurrir de forma aislada, pero a menudo está asociado con múltiples defectos de la línea media. Fue descrito por primera vez por Scott en 1958, tiene una incidencia de 1:50.000 nacidos vivos y se presenta con mayor frecuencia en mujeres. Su etiología es aún desconocida ${ }^{1,4}$. Pese a que la etiología se mantiene poco precisada, teorías plantean que se podría tratar de defectos que ocurren entre el día 35 y 38 en el útero, afectando las estructuras de la línea media de la cabeza, incluyendo los huesos del cráneo, el maxilar, la dentición, las vías respiratorias nasales $y$, en ocasiones, también del cerebro, junto con otras estructuras de la línea media del cuerpo. Anomalías asociadas (Tabla 1) incluyen defectos de la línea media y cavidad nasal, holoprosencefalia, microcefalia, retraso mental, enfermedad cardíaca congénita, fisura labiopalatal, hipopituitarismo, hipotelorismo, atresia esofágica y/o duodenal, órganos genitales ambiguos ${ }^{1,5-7}$.

El incisivo central no será visible en el nacimiento, pero puede ser reconocido por una prominencia en la línea media del alveolo maxilar,

Tabla 1. Defectos asociados a SICU

\begin{tabular}{|lr|}
\hline Defecto asociado a SICU & $\%$ \\
\hline Parto prematuro & 37 \\
Bajo peso al nacer & 37 \\
Estenosis apertura piriforme & 90 \\
Microcefalia & 33 \\
Estatura baja & 50 \\
Labio leporino/paladar hendido & 25 \\
Discapacidad intelectual & 50 \\
Alteración glándula pituitaria & $10-50$ \\
\hline
\end{tabular}


frenillo labial superior ausente y un labio superior en forma de arco. Un paladar en forma de $\mathrm{V}$ es también característico, debido a una cresta en la sutura media palatina. En el síndrome SICU aislado, sin asociación con otra alteración, puede no ser diagnosticado hasta que el incisivo central superior entra en erupción aproximadamente a 8 meses de edad. Dado que los recién nacidos son respiradores nasales obligados hasta 6-8 semanas de edad, SICU puede presentarse en el nacimiento con dificultad respiratoria debido a la obstrucción nasal amenazando la vida. Esto puede ser secundario a atresia de coanas o estenosis congénita de la apertura piriforme ${ }^{1}$.

La estenosis congénita de la apertura piriforme (ECAP) es una obstrucción de la vía aérea superior neonatal inusual, que fue descrita por primera vez en 1989. La verdadera incidencia es aún desconocida. Se estima que ECAP se produce con una frecuencia de alrededor de un cuarto a un tercio de la atresia de coanas, con una incidencia de 1 en 5.000 a 8.000 nacidos vivos, Esta anomalía puede ocurrir ya sea como un fenómeno aislado o asociado con defectos de la línea media como megaincisivo o incisivo único central maxilar, holoprosencefalia, agenesia hipofisiaria, disgenesia tiroidea y alteraciones cromosómicas entre otros. La presencia de anomalías asociadas debe ser estudiada mediante endoscopía nasal, resonancia magnética de cerebro, evaluación genética y endocrinológica-10.

La apertura piriforme es la parte más estrecha de la cavidad nasal delimitada superiormente por los huesos nasales, lateralmente por proceso nasal del maxilar superior e inferiormente por la unión del proceso horizontal del maxilar y la espina nasal anterior, cualquier reducción en su diámetro aumentará significativamente la resistencia de las vías respiratorias de acuerdo con la ley Pousielle (Figura 4), ésta permite calcular el flujo laminar estacionario lo que se relaciona con la resistencia de la vía aérea, en base al largo y radio de ésta así como la viscosidad del gas, teniendo como variable más importante el radio, el cual al estar elevado a la cuarta potencia, tiene un impacto tremendo sobre la resistencia. Mientras que la embriología y la etiología aún no están determinadas, se plantea que en el cuarto mes de desarrollo fetal existiría un crecimiento excesivo del proceso nasal del maxilar superior de forma bilateral, produciendo una disminución del calibre del orificio piriforme nasal, con la subsiguiente aparición de signos y síntomas de obstrucción $n^{9,11}$.

El diagnóstico de ECAP se debe sospechar cuando la dificultad respiratoria neonatal se combina con una dificultad para realizar la intubación nasal o pasar sondas nasogástricas. El diagnóstico se basa en la evaluación clínica, incluyendo la endoscopía nasal y, en especial, la tomografía computarizada. La imposibilidad de introducir un catéter 5 French $(F)(1.67 \mathrm{~mm}$ de diámetro exterior) y una apertura piriforme medida radiográficamente menor a 8-10 $\mathrm{mm}$ en un recién nacido de término son considerados como diagnóstico de una estenosis importante. Las formas más leves pueden presentarse como dificultad en la alimentación asociada con episodios de apnea o cianosis aliviados por medio del llanto (cianosis cíclico). El examen de confirmación se realiza mediante TC con una apertura piriforme menor a $8 \mathrm{~mm}$ (medido en la TC axial a nivel del meato inferior) en un recién nacido de término $0^{1,8,9,11}$.

Estudios afirman que megaincisivo acompaña a esta patología hasta en $75 \%$ de los casos 9 . El diagnóstico precoz es vital para el adecuado manejo de la enfermedad, el tratamiento puede ser conservador o quirúrgico dependiendo de la gravedad de los síntomas. El enfoque conservador inicial se realiza usando inhaladores nasales

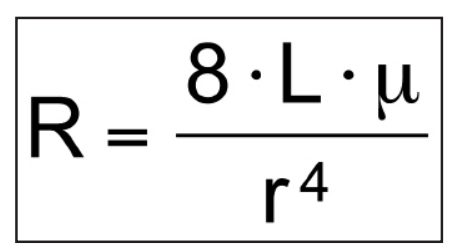

Figura 4. Ley de Pousielle donde L: es el largo del tubo, $\mu$ : viscosidad del gas , r: radio del tubo. 
de corticosteroides y oximetazolina, durante los períodos de mayor obstrucción. Criterios para el manejo quirúrgico incluyen la apnea del sueño, la intubación repetida o el fracaso de la extubación, dificultades frecuentes en la alimentación asociada con cianosis y el fracaso de los métodos conservadores ${ }^{1}$.

En general el tratamiento de ECAP depende primero del pronóstico global del paciente y segundo de la gravedad de la obstrucción. Los pacientes con mal pronóstico deben ser manejados de forma conservadora, manteniendo una vía respiratoria orotraqueal. En los pacientes con buen pronóstico, el tratamiento conservador es de elección para aquellos con obstrucción leve y la corrección quirúrgica para aquellos pacientes con obstrucción completa ${ }^{8}$.

El manejo inicial de esta condición implica la creación de una vía aérea segura mediante la colocación de chupete McGovern o intubación endotraqueal, con un seguimiento adecuado en la unidad de cuidados intensivos hasta que se haya establecido la causa exacta y la severidad de la obstrucción nasal. En los casos de ECAP leve, un enfoque no quirúrgico implica la colocación de stents en la cavidad nasal y el uso de descongestionantes locales. Sin embargo, las pequeñas dimensiones de los stents nasales pueden conducir a la oclusión y hacer la limpieza diaria muy difícil, lo que aumenta el riesgo de obstrucción y lesiones de los tejidos blandos durante la limpieza y reposición ${ }^{12}$.

En los casos de estenosis moderada o grave, el enfoque es quirúrgico y consiste en la ampliación de la apertura piriforme a través de un abordaje sublabial endo-oral. La disección y el fresado deben realizarse de forma anterior al cornete inferior, para evitar el daño del conducto nasolagrimal. Este método es seguro y permite una buena exposición de campo, evita daños en los tejidos blandos nasolabiales, en los brotes dentarios, conductos nasolagrimales y no causa cicatrices visibles. El crecimiento óseo excesivo se debe quitar con el fin de obtener un ancho del orificio piriforme $>11 \mathrm{~mm}$. Algunos autores también abogan por una reducción del extremo anterior del cornete inferior para crear una vía respiratoria adecuada. En la mayoría de los casos se utilizan stents nasales después de la cirugía para ayudar a mantener la vía aérea nasal y prevenir la reestenosis. Se recomienda retirarlos entre 1 y 4 semanas ${ }^{1,11}$.

Las posibles complicaciones de un abordaje quirúrgico incluyen necrosis columelar, que ha sido descrita en uno de cada 20 casos quirúrgicos, el desarrollo de sinequias y ulceración septal con perforación septal ${ }^{8}$.

SICU se reconoce como un posible predictor de holoprosencefalia (HPE) de diverso grado. Por lo tanto, el consejo genético es obligatorio en estos casos. El diente mismo SICU es un verdadero diente incisivo central, pero no es un diente supernumerario. Desarrolla y estalla precisamente en la línea media del arco dental maxilar. Sólo en casos muy raros, SICU no contribuyen a la estenosis de la apertura piriforme, de ser así, se trata principalmente de un problema estético y el tratamiento es gestionado por ortodoncia, mediante prótesis y el tratamiento quirúrgico por vía oral. Alternativamente, se puede dejar sin tratar8.

La deleción del cromosoma 7 y 8 están asociados con SICU; estos son los cromosomas, que portan los genes para HPE y por lo tanto SICU se considera como un marcador o microformas de holoprosencefalia. Por lo tanto, la aparición de SICU con estenosis de la apertura piriforme obliga a realizar una resonancia magnética cerebral para descartar las características asociadas de holoprosencefalia y trastornos pituitarios ${ }^{9,13}$.

\section{CONCLUSIÓN}

La ECAP es una causa rara y potencialmente letal de obstrucción de vías respiratorias en los recién nacidos. Su origen se encuentra en anomalías del desarrollo de estructuras de la línea media craneofacial, de etiología desconocida, por lo que también puede existir un megaincisivo o incisivo central medio solitario maxilar, 0 presentar alteraciones endocrinas, por lo que se hace imprescindible realizar una monitorización del eje hipotálamo-hipofisario. ECAP debe considerarse siempre en el diagnóstico diferencial en los recién nacidos con signos de obstrucción nasal grave, asociada con una dificultad en pasar un pequeño catéter a través de la válvula nasal anterior. EI diagnóstico puede realizarse con la clínica y la exploración de la vía aérea superior, pero es el TAC 
el que nos aporta información definitiva y nos ayuda a plantear la cirugía, en caso necesario. Según la gravedad de la clínica, el tratamiento será variable,

\section{BIBLIOGRAFÍA}

1. BLACKMORE K, Wynne DM. A case of solitary median maxillary central incisor (SMMCI) syndrome with bilateral pyriform aperture stenosis and choanal atresia. Int J Pediatr Otorhinolaryngol 2010; 74(8): 967-9.

2. Szakszon K, Felszeghy E, Csízy I, Józsa T, Káposzta R, Balogh E, et al. Endocrine and anatomical findings in a case of Solitary Median Maxillary Central Incisor Syndrome. Eur J Med Genet 2012; 55(2): 109-1.

3. Gimeno-Hernández J, Iglesias-Moreno MC, GómezSerrano M, Рoch-Broto J. Estenosis congénita del orificio piriforme y megaincisivo central único. Acta Otorrinolaringológica Esp 2010; 61(6): 455-8.

4. Shilpa G, Nuvvula S, Gokhale N, Yamini V. Concomitant solitary median maxillary central incisor and fused right mandibular incisor in primary dentition. Contemp Clin Dent 2012; 3(Suppl 2): S203-5.

5. Giannopoulou EZ, Rohrer T, Hoffmann P, Yilmaz U, Gortner L, Meyer S. Solitary Median Maxillary Central Incisor. J Pediatr 2015; 167(3): 770-770.e1.

6. Van Dijk FS, van ThuiJl HF, Wermeskerken A, van RIJn RR, CobBen JM. Solitary median maxillary central incisor and congenital nasal pyriform aperture stenosis combined with asymmetric crying facies and postaxial lower limb reduction desde una actitud expectante con aplicación de medidas conservadoras hasta cirugía como último recurso. defects: A unique combination of features. Eur $J$ Med Genet 2011; 54(3): 284-6.

7. HALL RK. Solitary median maxillary central incisor (SMMCI) syndrome. Orphanet J Rare Dis 2006; 1: 12.

8. Günther L, Sari-Rieger A, Jablonka K, Rustemeyer J. Clinical course and implications of congenital nasal pyriform stenosis and solitary median maxillary central incisor in a newborn: a case report. J Med Case Reports 2014; 8: 215.

9. Kumar nd, Kumaran aA, Balaju S, Anupriya R. Congenital nasal pyriform aperture stenosis: A rare cause of respiratory distress in newborn. $J$ Clin Neonatol 2015; 4(3): 203.

10. Madrigal M, Heras P de las, Gil-Carcedo E, Enterría Á, Acuña M, GiL-Carcedo LM. Estenosis congénita de la apertura piriforme: forma inusual de obstrucción nasal. Rev Esp Cir Oral Maxilofac 2014; 36(3): 129-31.

11. Sesenna E, Leporati M, Brevi B, Oretti G, Ferri A. Congenital nasal pyriform aperture stenosis: diagnosis and management. Ital J Pediatr 2012; 38: 28.

12. Cuestas G, José G, Demarchi V, Razetti J, Boccio C. Obstrucción nasal congénita por estenosis de la apertura piriforme. Serie de casos. An Pediatría 2015; 82(1): e44-7.

13. Machado E, Machado P, Grehs B, Grehs RA. Solitary median maxillary central incisor syndrome: case report. Dent Press J Orthod 2010; 15(4): 55-61.

Dirección: Rodolfo Nazar S. 